\title{
Irreversible Capital Accumulation with Economic Impact
}

\author{
Hessah Al Motairi ${ }^{1} \cdot$ Mihail Zervos $^{2}$
}

Published online: 21 March 2016

(C) The Author(s) 2016. This article is published with open access at Springerlink.com

\begin{abstract}
We consider an irreversible capacity expansion model in which additional investment has a strictly negative effect on the value of an underlying stochastic economic indicator. The associated optimisation problem takes the form of a singular stochastic control problem that admits an explicit solution. A special characteristic of this stochastic control problem is that changes of the state process due to control action depend on the state process itself in a proportional way.
\end{abstract}

Keywords Singular stochastic control · Variational inequality · Capacity expansion · Irreversible investment

\section{Introduction}

A standard capacity expansion model, which is a special case of the model studied by Kobila [34], can be described as follows. We model market uncertainty by means of the geometric Brownian motion given by

$$
d X_{t}^{0}=b X_{t}^{0} d t+\sqrt{2} \sigma X_{t}^{0} d W_{t}, \quad X_{0}^{0}=x>0,
$$

for some constants $b$ and $\sigma \neq 0$, where $W$ is a standard one-dimensional Brownian motion. The random variable $X_{t}^{0}$ can represent an economic indicator such as the price

Mihail Zervos

mihalis.zervos@gmail.com

Hessah Al Motairi

almotairi.h@gmail.com

1 Department of Mathematics, College of Science, Kuwait University, Kuwait City, Kuwait

2 Department of Mathematics, London School of Economics, Houghton Street, London WC2A 2AE, UK 
of or the demand for one unit of a given investment project's output at time $t$. The firm behind the project can invest additional capital at proportional costs at any time, but cannot disinvest from the project. We denote by $y$ the project's initial capital at time 0 and by $\zeta_{t}$ the total additional capital invested by time $t$. We assume that there is no capital depreciation, so the total capital invested at time $t$ is

$$
Y_{t}=y+\zeta_{t}, \quad Y_{0}=y \geq 0
$$

The investor's objective is to maximise the total expected discounted payoff resulting from the project's management, which is given by the performance index

$$
J_{x, y}^{0}(\zeta)=\mathbb{E}\left[\int_{0}^{\infty} e^{-r t} h\left(X_{t}^{0}, Y_{t}\right) d t-K \int_{[0, \infty[} e^{-r t} d \zeta_{t}\right]
$$

over all capacity expansion strategies $\zeta$. The discounting rate $r>0$ and the cost of each additional unit of capital $K>0$ are constants, while $h$ is an appropriate running payoff function.

Under suitable assumptions on the problem data, the solution to this stochastic control problem is characterised by a threshold given by a strictly increasing freeboundary function $G^{0}: \mathbb{R}_{+} \rightarrow \mathbb{R}_{+}$. In the special case that arises when $h(x, y)=$ $x^{\alpha} y^{\beta}$, for some $\alpha>0$ and $\left.\beta \in\right] 0,1$, namely, when $h$ is a so-called Cobb-Douglas production function,

$$
G^{0}(y)=\left(\frac{r K(\alpha-m)}{-m \beta}\right)^{\frac{1}{\alpha}} y^{\frac{1-\beta}{\alpha}} \text { for } y \geq 0,
$$

where $m<0$ is an appropriate constant. If the initial condition $(x, y)$ is strictly below the graph of the function $G^{0}$ in the $x-y$ plane, then it is optimal to invest so that the joint process $\left(X^{0}, Y\right)$ has a jump at time 0 that positions it in the graph of $G^{0}$. Otherwise, it is optimal to take minimal action so that the state process $\left(X^{0}, Y\right)$ does not fall below the graph of $G^{0}$, which amounts to reflecting it in $G^{0}$ in the positive $y$-direction.

Irreversible capacity expansion models have attracted considerable interest and can be traced back to Manne [38] (see Van Mieghem [47] for a survey). More relevant to this paper models have been studied by several authors in the economics literature: see Dixit and Pindyck [17, Chapter 11] and references therein. Related models that have been studied in the mathematics literature include Davis, Dempster, Sethi and Vermes [13], Arntzen [4], Øksendal [42], Wang [48], Chiarolla and Haussmann [11], Bank [6], Alvarez [2,3], Løkka and Zervos [35], Steg [45], Chiarolla and Ferrari [9], De Angelis, Federico and Ferrari [15], and references therein. Furthermore, capacity expansion models with costly reversibility were introduced by Abel and Eberly [1], and were further studied by Guo and Pham [22], Merhi and Zervos [40], Guo and Tomecek [23,24], Guo, Kaminsky, Tomecek and Yuen [21], Løkka and Zervos [36], De Angelis and Ferrari [16], and Federico and Pham [19].

In the model that we have briefly discussed above, additional investment does not influence the underlying economic indicator, which is unrealistic if one considers supply and demand issues. The nature of the optimal strategy is such that, if $b<\frac{1}{2} \sigma^{2}$, 
then $\lim _{t \rightarrow \infty} X_{t}^{0}=0$ and the investment's maximal optimal capacity level remains finite for realistic choices of the problem data. On the other hand, if $b \geq \frac{1}{2} \sigma^{2}$, then $\lim \sup _{t \rightarrow \infty} X_{t}^{0}=\infty$ and the optimal capacity level typically converges to $\infty$ as $t \rightarrow \infty$.

The model that we study here assumes that additional investment has a strictly negative effect on the value of the underlying economic indicator process $X$. We assume that increasing the project's capacity by a very small amount $\Delta \zeta_{t}=\varepsilon$ at time $t$ affects the process $X$ linearly, namely,

$$
\Delta X_{t} \equiv X_{t+}-X_{t}=-c \varepsilon X_{t} \Rightarrow X_{t+}=(1-c \varepsilon) X_{t} \simeq e^{-c \varepsilon} X_{t}
$$

for some constant $c>0$, where we have taken $X$ to be càglàd. Furthermore, we assume that increasing the project's capacity by an amount $\Delta \zeta_{t}>0$ at time $t$ has the same effect on the process $X$ as increasing the project's capacity $N$ times infinitesimally close to each other by an amount $\Delta \zeta_{t} / N$ for every choice of $N$, which gives rise to the identities

$$
X_{t+}=e^{-c\left(\Delta \zeta_{t} / N\right) N} X_{t}=e^{-c \Delta \zeta_{t}} X_{t} .
$$

These considerations suggest the modelling of market uncertainty by the solution to the SDE

$$
d X_{t}=b X_{t} d t-X_{t} \circ d \zeta_{t}+\sqrt{2} \sigma X_{t} d W_{t}, \quad X_{0}=x>0
$$

where

$$
\int_{0}^{t} X_{s} \circ d \zeta_{s}=c \int_{0}^{t} X_{s} d \zeta_{s}^{\mathrm{c}}+\sum_{0 \leq s<t} X_{t}\left(1-e^{-c \Delta \zeta_{t}}\right),
$$

in which expression, $\zeta^{\mathrm{c}}$ denotes the continuous part of the increasing process $\zeta$. At this point, it is worth noting that Guo and Zervos [25] have considered the same state dynamics in the optimal execution problem that they study. The objective is to maximise over all admissible capacity expansion strategies $\zeta$ the performance criterion

$$
J_{x, y}(\zeta)=\mathbb{E}\left[\int_{0}^{\infty} e^{-r t} h\left(X_{t}, Y_{t}\right) d t-K \int_{[0, \infty[} e^{-r t} d \zeta_{t}\right]
$$

where $r, K>0$ are constants and the running payoff function $h$ satisfies Assumption 1 in the next section.

The solution to this problem is again characterised by a threshold defined by a strictly increasing free-boundary function $G$. Informally, the optimal strategy can be described as the one in the problem defined by (1)-(3). However, reflection in the free-boundary $G$ is oblong rather than in the positive $y$-direction (see Figs. 1, 2, 3). Furthermore, the negative effect that additional investment has on the underlying economic indicator $X$ results in a maximal optimal capacity level that is bounded in cases of special interest, such as the ones arising, e.g., when the running payoff function $h$ is a Cobb-Douglas production function (see Example 2).

From a stochastic control theoretic perspective, the problem that we solve has the features of singular stochastic control, which was introduced by Bather and Chernoff [7] who considered a simplified model of spaceship control. In their seminal paper, 


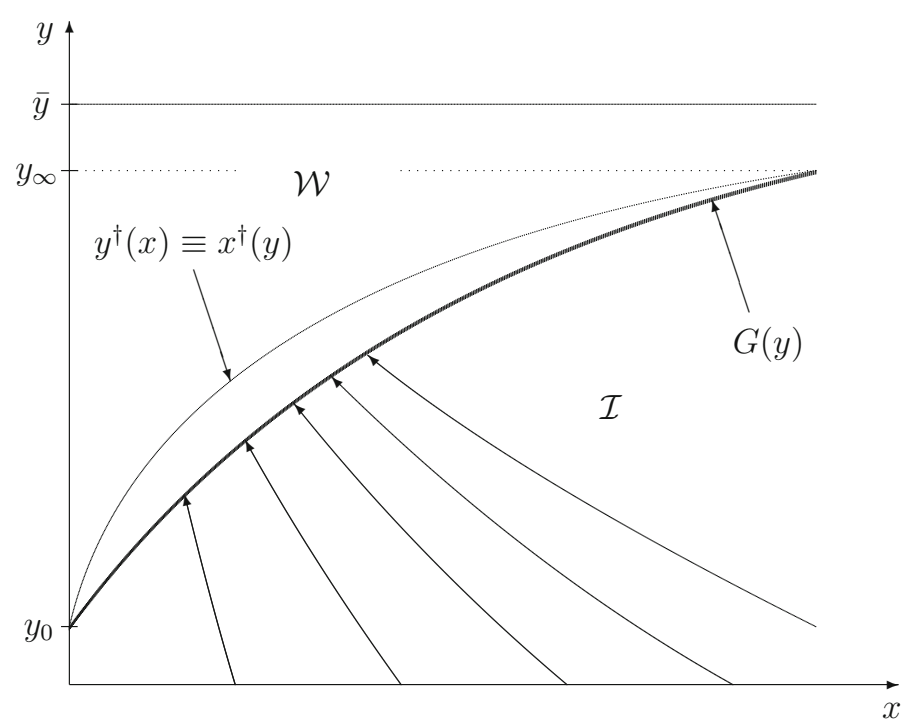

Fig. 1 Graph of the free-boundary function $G$ in the general context

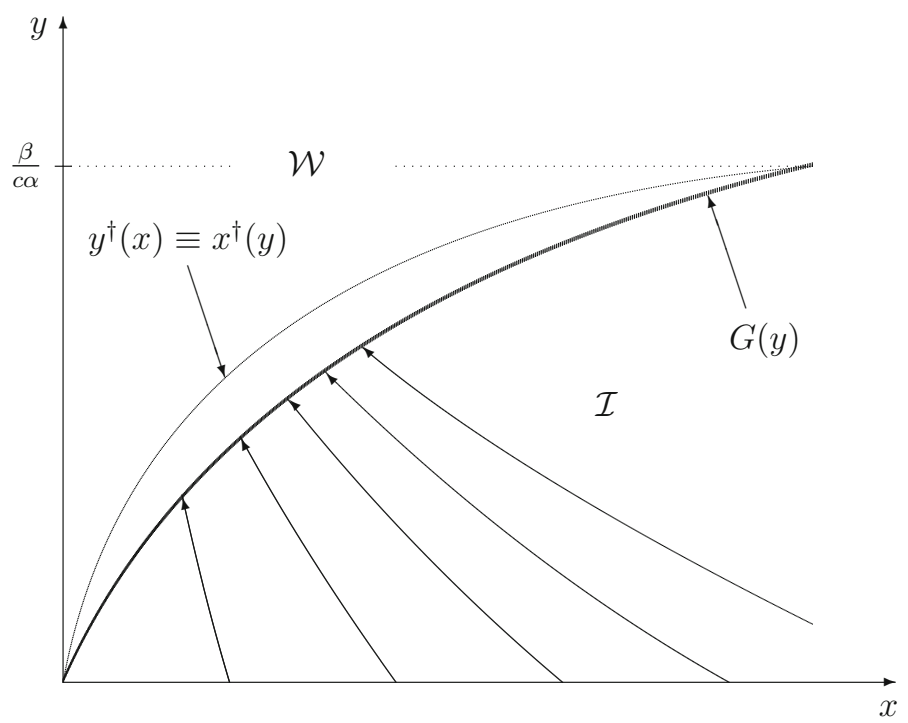

Fig. 2 Graph of $G$ when $h$ is a Cobb-Douglas function with $\beta \in] 0,1[$

Beneš, Shepp and Witsenhausen [8] were the first to solve rigorously an example of a finite-fuel singular control problem. Since then, the area has attracted considerable interest in the literature. Apart from references that we have discussed in the context of capacity expansion models, Bahlali et al. [5] Chiarolla and Haussmann [10], Chow, Menaldi and Robin [12], Davis and Zervos [14], Fleming and Soner [20, Chapter VIII], 


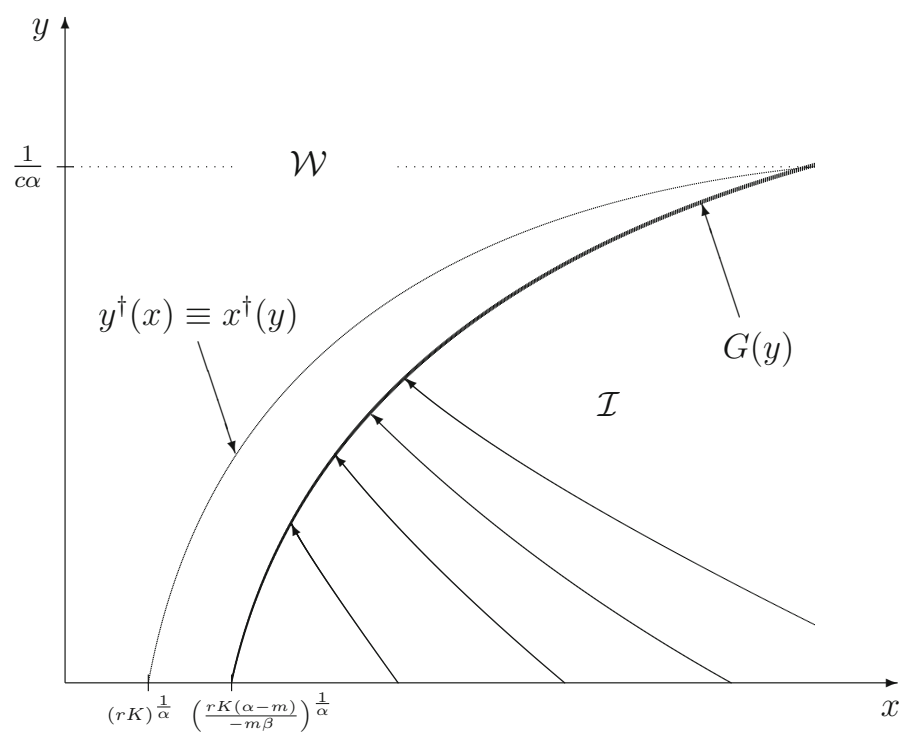

Fig. 3 Graph of $G$ when $h$ is a Cobb-Douglas function with $\beta=1$

Haussmann and Suo [27, 28], Harrison and Taksar [26], Jack, Johnson and Zervos [29], Jacka [30,31], Karatzas [32], Ma [37], Menaldi and Robin [39], Øksendal [42], Shreve et al. [43], Soner and Shreve [44], Sun [46] and Zhu [49], provide an alphabetically ordered list of further contributions.

In the references discussed above, the controlled process affects the state dynamics in a purely additive way: the change of the state process due to control action does not depend on the state process itself. Singular stochastic control models in which changes of the state process due to control action may depend on the state process were introduced and studied by Dufour and Miller [18] and Motta and Sartori [41]. To the best of our knowledge, problems with state dynamics such as the ones given by (4)-(5) have not been considered in the literature before. Furthermore, the problem that we solve is the very first one in the singular stochastic control literature that involves control action that does not affect the state dynamics in a purely additive way and admits an explicit solution (see also Remark 1 in the next section).

\section{Problem Formulation and Assumptions}

We fix a probability space $(\Omega, \mathcal{F}, \mathbb{P})$ equipped with a filtration $\left(\mathcal{F}_{t}\right)$ satisfying the usual conditions of right continuity and augmentation by $\mathbb{P}$-negligible sets, and carrying a standard one-dimensional $\left(\mathcal{F}_{t}\right)$-Brownian motion $W$. We denote by $\mathcal{Z}$ the family of all càglàd $\left(\mathcal{F}_{t}\right)$-adapted increasing process $\zeta$ such that $\zeta_{0}=0$.

The state space of the control problem that we study is defined by

$$
\mathcal{S}=\left\{(x, y) \in \mathbb{R}^{2} \mid x>0 \text { and } 0 \leq y \leq \bar{y}\right\},
$$


where $\bar{y} \in] 0, \infty]$ is the maximal capital that can be invested in the project, namely, the maximum capacity level that can be achieved. Given a capacity expansion processes $\zeta \in \mathcal{Z}$, we consider the capacity process $Y$ defined by (2) and the economic indicator process $X$ given by (4)-(5). Using Itô's formula, we can verify that

$$
X_{t}=X_{t}^{0} e^{-c \zeta_{t}^{\mathrm{c}}} \prod_{0 \leq s<t}\left(1-e^{-c \Delta \zeta_{t}}\right)=X_{t}^{0} e^{-c \zeta_{t}}
$$

where $X^{0}$ is the geometric Brownian motion defined by (1).

Definition 1 The set $\mathcal{A}$ of all admissible capacity expansion strategies is the family of all processes $\zeta \in \mathcal{Z}$ such that

$$
\mathbb{E}\left[\int_{[0, \infty[} e^{-r t} d \zeta_{t}\right]<\infty
$$

The objective of the control problem is to maximise the performance index $J_{x, y}$ defined by (6) over all admissible strategies $\zeta \in \mathcal{A}$, for each initial condition $(x, y) \in \mathcal{S}$. Accordingly, we define the problem's value function $v$ by

$$
v(x, y)=\sup _{\zeta \in \mathcal{A}} J_{x, y}(\zeta), \quad \text { for }(x, y) \in \mathcal{S}
$$

Remark 1 In view of (7), we can see that the stochastic optimisation problem we solve is equivalent to maximising

$$
J_{x, y}(\zeta)=\mathbb{E}\left[\int_{0}^{\infty} e^{-r t} h\left(e^{c y} X_{t}^{0} e^{-c Y_{t}}, Y_{t}\right) d t-K \int_{[0, \infty[} e^{-r t} d \zeta_{t}\right]
$$

over all admissible strategies $\zeta \in \mathcal{A}$, where the dynamics of the state process $\left(X^{0}, Y\right)$ are given by (1)-(2). At first glance, this observation puts us in the context of the standard singular stochastic control theory because control action affects the dynamics of $\left(X^{0}, Y\right)$ in a purely additive way. However, such a reformulation is of limited theoretical value because the problem's initial condition $y$ enters non-trivially in the description of the performance criterion, which is a situation that is typically associated with time-inconsistent control problems.

Our analysis involves the general solution to the second order Euler's ODE

$$
\sigma^{2} x^{2} u^{\prime \prime}(x)+b x u^{\prime}(x)-r u(x)=0,
$$

which is given by

$$
u(x)=A x^{n}+B x^{m},
$$


for some $A, B \in \mathbb{R}$, where the constants $m<0<n$ are the solutions to the quadratic equation

$$
\sigma^{2} \lambda^{2}+\left(b-\sigma^{2}\right) \lambda-r=0
$$

given by

$$
m, n=\frac{-\left(b-\sigma^{2}\right) \pm \sqrt{\left(b-\sigma^{2}\right)^{2}+4 \sigma^{2} r}}{2 \sigma^{2}} .
$$

Our analysis also involves the function $H$ defined by

$$
\left.H(x, y)=h_{y}(x, y)-c x h_{x}(x, y)-r K, \quad \text { for } x>0 \text { and } y \in\right] 0, \bar{y}[\text {. }
$$

This function has a natural economic interpretation. Indeed, increasing capacity by a small amount $\varepsilon>0$ causes the joint process $(X, Y)$ to jump from a value $(x, y)$ to the value $(x-c x \varepsilon, y+\varepsilon)$. Noting that

$h(x-c x \varepsilon, y+\varepsilon)-h(x, y) \simeq\left[h_{y}(x, y)-c x h_{x}(x, y)\right] \varepsilon$ and $K=\int_{0}^{\infty} e^{-r t} r K d t$,

we can see that $H(x, y)$ represents the project's marginal running payoff rate in excess of the marginal cost of capital rate. In view of standard economics theory, this interpretation suggests that (a) the function $H(\cdot, y)$ should be increasing for all $y \geq 0$ because higher values of the underlying economic indicator $X$, which models the price of or the demand for one unit of the project's output, should reflect higher values of marginal running payoff, and (b) the function $H(x, \cdot)$ should be decreasing for all $x>0$ because the project's payoff rate should be concave in the volume of its output due to the balancing of supply and demand. These observations suggest requirements (17)-(19) in the following assumption. In fact, the conditions reflected by (17)-(19) are much weaker than the ones suggested by the above considerations. However, the relaxations involved present no added complications in our analysis whatsoever. The underlying economics theory also suggests that the running payoff function $h$ should be increasing in the value of the underlying economic indicator $X$ for each fixed value of the project's capacity, which is captured by condition (14). The rest of the conditions appearing in the following assumption, which is admittedly rather long to state, are of a purely technical nature. It is worth noting that (15) is equivalent to the probabilistic condition

$$
\mathbb{E}\left[\int_{0}^{\infty} e^{-r t}\left|h\left(X_{t}^{0}, y\right)\right| d t\right]<\infty \text { for all } x>0 \text { and } y \in[0, \bar{y}] \cap \mathbb{R}
$$

(see (77)-(78) in Appendix 2).

Assumption 1 The constants $r, K$ are strictly positive, the function $h$ is $C^{3}$,

$$
\begin{gathered}
h(\cdot, y) \text { is increasing for all } y \in[0, \bar{y}] \cap \mathbb{R} \\
\int_{0}^{x} s^{-m-1}|h(s, y)| d s+\int_{x}^{\infty} s^{-n-1}|h(s, y)| d s<\infty \text { for all } x>0 \text { and } y \in[0, \bar{y}] \cap \mathbb{R}
\end{gathered}
$$


There exists a point $x_{0} \geq 0$ and a continuous strictly increasing function $y^{\dagger}$ : ]$x_{0}, \infty\left[\rightarrow \mathbb{R}_{+}\right.$such that

$$
\begin{array}{r}
0 \leq y_{0}:=\lim _{x \downarrow x_{0}} y^{\dagger}(x)<\lim _{x \rightarrow \infty} y^{\dagger}(x)=: y_{\infty} \leq \bar{y}, \quad y_{0}=0 \text { if } x_{0}>0, \\
H(x, y) \begin{cases}<0, & \text { if }(x, y) \in \mathcal{H}_{-}, \\
=0, & \text { if }(x, y) \in \mathcal{S} \backslash\left(\mathcal{H}_{-} \cup \mathcal{H}_{+}\right), \\
>0, & \text { if }(x, y) \in \mathcal{H}_{+},\end{cases} \\
\left.\liminf _{x \rightarrow \infty} H(x, y)>0 \text { for all } y \in\right] y_{0}, y_{\infty}[,
\end{array}
$$

the function $H(x, \cdot)$ is strictly decreasing for all $y \in] y_{0}, y_{\infty}[$,

where

$$
\begin{array}{r}
\mathcal{H}_{-}=\left\{(x, y) \in \mathcal{S} \mid x \leq x_{0} \text { or } x>x_{0} \text { and } y>y^{\dagger}(x)\right\} \\
\mathcal{H}_{+}=\left\{(x, y) \in \mathcal{S} \mid x>x_{0} \text { and } y<y^{\dagger}(x)\right\}
\end{array}
$$

Also, there exist a decreasing function $\Psi:] y_{0}, y_{\infty}[\rightarrow] 0, \infty[$ such that $\lim _{y \downarrow 0} \Psi(y)<\infty$ if $x_{0}>0$ as well as constants $C_{0}>0$ and $\left.\vartheta \in\right] 0, n[$ such that

$$
\begin{array}{r}
-C_{0}(1+y) \leq h(x, y) \leq C_{0}(1+y)\left(1+x^{n-\vartheta}\right) \quad \text { for all }(x, y) \in \mathcal{S} \\
\left.H(x, y) \leq \Psi(y)\left(1+x^{n-\vartheta}\right) \text { for all } x>0 \text { and } y \in\right] 0, \bar{y}[
\end{array}
$$

We denote by $x^{\dagger}$ the inverse of the function $y^{\dagger}$ that is defined by

$$
x^{\dagger}(y)= \begin{cases}0, & \text { if } 0 \leq y<y_{0} \\ \left(y^{\dagger}\right)^{-1}(x), & \text { if } y_{0} \leq y<y_{\infty} \\ \infty, & \text { if } y_{\infty} \leq y<\bar{y}\end{cases}
$$

Example 1 Suppose that $\bar{y}=\infty$ and $h$ is a so-called Cobb-Douglas function, given by

$$
h(x, y)=x^{\alpha} y^{\beta}, \quad \text { for }(x, y) \in \mathcal{S},
$$

where $\alpha \in] 0, n[$ and $\beta \in] 0,1]$ are constants. In this case, we can check that

$$
H(x, y)=\left(\beta y^{-1}-c \alpha\right) x^{\alpha} y^{\beta}-r K .
$$

If we define

$$
y_{0}=0, \quad y_{\infty}=\frac{\beta}{c \alpha} \quad \text { and } \quad x_{0}= \begin{cases}(r K)^{1 / \alpha}, & \text { if } \beta=1, \\ 0, & \text { if } \beta \in] 0,1[\end{cases}
$$


then we can see that the calculations

$$
\begin{aligned}
\frac{\partial H(x, y)}{\partial x} & =\alpha\left(\beta y^{-1}-c \alpha\right) x^{\alpha-1} y^{\beta} \begin{cases}>0 & \text { for all } y \in] y_{0}, y_{\infty}[, \\
<0 & \text { for all } y \geq y_{\infty},\end{cases} \\
\lim _{x \downarrow 0} H(x, y) & =-r K<0 \text { for all } y>0 \text { and } \lim _{x \rightarrow \infty} H(x, y)
\end{aligned}
$$

imply that there exists a unique function $\left.y^{\dagger}:\right] x_{0}, \infty\left[\rightarrow \mathbb{R}_{+}\right.$such that (16)-(17) hold true. Furthermore, differentiating the identity $H\left(x, y^{\dagger}(x)\right)=0$ with respect to $x$, we can see that

$$
\left.\dot{y}^{\dagger}(x)=\frac{\alpha y(\beta-c \alpha y)}{\beta x[(1-\beta)+c \alpha y]}>0 \text { for all } y \in\right] y_{0}, y_{\infty}[,
$$

so $y^{\dagger}$ is indeed strictly increasing. Also, it is straightforward to check that (19)-(18) and (20)-(21) are all satisfied for $\vartheta=n-\alpha$ and

$$
\Psi(y)= \begin{cases}1, & \text { if } \beta=1 \\ y^{-(1-\beta)}, & \text { if } \beta \in] 0,1[\end{cases}
$$

\section{The Solution to the Control Problem}

We solve the stochastic control problem that we consider by constructing an appropriate classical solution $w: \mathcal{S} \rightarrow \mathbb{R}$ to the Hamilton-Jacobi-Bellman (HJB) equation

$$
\begin{aligned}
\max \left\{\sigma^{2} x^{2} w_{x x}(x, y)+b x w_{x}(x, y)-r w(x, y)+h(x, y),\right. & \\
\left.w_{y}(x, y)-c x w_{x}(x, y)-K\right\} & =0, \quad(x, y) \in \mathcal{S},
\end{aligned}
$$

where $w_{y}(x, 0)=\lim _{y \downarrow} w_{y}(x, y)$. To obtain qualitative understanding of this equation, we consider the following heuristic arguments. At time 0 , the project's management has two options. The first one is to wait for a short time $\Delta t$ and then continue optimally. Bellman's principle of optimality implies that this option, which is not necessarily optimal, is associated with the inequality

$$
v(x, y) \geq E\left[\int_{0}^{\Delta t} e^{-r t} h\left(X_{t}^{0}, y\right) d t+e^{-r \Delta t} v\left(X_{\Delta t}^{0}, y\right)\right] .
$$


Applying Itô's formula to the second term in the expectation, and dividing by $\Delta t$ before letting $\Delta t \downarrow 0$, we obtain

$$
\sigma^{2} x^{2} v_{x x}(x, y)+b x v_{x}(x, y)-r v(x, y)+h(x, y) \leq 0 .
$$

The second option is to increase capacity by $\varepsilon>0$, and then continue optimally. This action is associated with the inequality

$$
v(x, y) \geq v(x-c x \varepsilon, y+\varepsilon)-K \varepsilon .
$$

Rearranging terms and letting $\varepsilon \downarrow 0$, we obtain

$$
v_{y}(x, y)-c x v_{x}(x, y)-K \leq 0 .
$$

Furthermore, the Markovian character of the problem implies that one of these options should be optimal and one of (25), (26) should hold with equality at any point in the state space $\mathcal{S}$. It follows that the problem's value function $v$ should identify with an appropriate solution $w$ to the HJB equation (24).

To construct the solution $w$ to (24) that identifies with the value function $v$, we first consider the existence of a strictly increasing function $G:] y_{0}, y_{\infty}[\rightarrow] 0, \infty[$ that partitions the state space $\mathcal{S}$ into two regions, the "waiting" region $\mathcal{W}$ and the "investment" region $\mathcal{I}$ defined by

$$
\begin{aligned}
\mathcal{W}= & \left\{(x, 0) \mid 0<x \leq x_{0} \text { if } x_{0}>0\right\} \\
& \cup\{(x, y) \mid y \in] y_{0}, y_{\infty}[\text { and } 0<x \leq G(y)\} \\
& \cup\left\{(x, y) \mid x>0 \text { and } y \in\left[y_{\infty}, \bar{y}\right] \cap \mathbb{R}\right\}, \\
\mathcal{I}= & \left\{(x, 0) \mid x>x_{0} \text { if } x_{0}>0\right\} \\
& \cup\left\{(x, y) \mid x>0 \text { and } y \in\left[0, y_{0}\right] \text { if } y_{0}>0\right\} \\
& \cup\{(x, y) \mid y \in] y_{0}, y_{\infty}[\text { and } x>G(y)\} .
\end{aligned}
$$

In view of the interpretation of the function $H$ defined by (13) as the project's marginal running payoff rate in excess of the marginal cost of capital rate, which we have discussed in the previous section, we can see that increasing capacity cannot be optimal whenever the state process takes values $(x, y) \in \mathcal{S}$ such that $H(x, y)<0$. This observation, (17) in Assumption 1 and (22) suggest that the inequality

$$
\left.G(y)<x^{\dagger}(y) \text { for all } y \in\right] y_{0}, y_{\infty}[
$$

should hold true. Figures 1, 2, and 3 depict possible configurations of the waiting and the investment regions.

Inside the region $\mathcal{W}$, the heuristic arguments that we have briefly discussed above suggest that $w$ should satisfy the differential equation

$$
\sigma^{2} x^{2} w_{x x}(x, y)+b x w_{x}(x, y)-r w(x, y)+h(x, y)=0 .
$$


In light of the theory that we review in Appendix 2 and the intuitive idea that the value function should remain bounded as $x \downarrow 0$, every relevant solution to this ODE is given by

$$
w(x, y)=A(y) x^{n}+R(x, y),
$$

for some function $A$, where $n$ is given by $(12)$ and $R(\cdot, y)$ is defined by (79) for $k=h(\cdot, y)$, namely,

$$
R(x, y)=\frac{1}{\sigma^{2}(n-m)}\left[x^{m} \int_{0}^{x} s^{-m-1} h(s, y) d s+x^{n} \int_{x}^{\infty} s^{-n-1} h(s, y) d s\right]
$$

On the other hand, $w$ should satisfy

$$
w_{y}(x, y)-c x w_{x}(x, y)=K, \quad \text { for }(x, y) \in \mathcal{I}
$$

which implies that

$$
w_{y x}(x, y)-c x w_{x x}(x, y)-c w_{x}(x, y)=0, \quad \text { for }(x, y) \in \mathcal{I} .
$$

To determine $A$ and $G$, we postulate that $w$ is $C^{2,1}$, in particular, along the freeboundary $G$. Such a requirement and (28)-(31) yield the system of equations

$$
\begin{aligned}
{[\dot{A}(y)-n c A(y)] G^{n}(y)=} & -\left[R_{y}(G(y), y)-c G(y) R_{x}(G(y), y)-K\right], \\
{[\dot{A}(y)-n c A(y)] G^{n}(y)=} & -\frac{G(y)}{n}\left[R_{y x}(G(y), y)\right. \\
& \left.-c G(y) R_{x x}(G(y), y)-c R_{x}(G(y), y)\right] .
\end{aligned}
$$

In view of the definition (29) of $R$, the associated expression (84) for the function $x \mapsto x R_{x}(x, y)$ and (83), we can see that this system is equivalent to

$$
\dot{A}(y)=n c A(y)-\frac{1}{\sigma^{2}(n-m)} \int_{G(y)}^{\infty} s^{-n-1} H(s, y) d s,
$$

where $H$ is defined by (13) and

$$
q(x, y)=\int_{0}^{x} s^{-m-1} H(s, y) d s .
$$


We can also check that the solution to (35) is given by

$$
A(y)=\frac{e^{c n y}}{\sigma^{2}(n-m)} \int_{y}^{y_{\infty}} e^{-c n u} \int_{G(u)}^{\infty} s^{-n-1} H(s, u) d s d u, \quad \text { for } y_{0}<y<y_{\infty},
$$

if the integrals converge.

The following result, the proof of which we develop in Appendix 1, is concerned with the solution to the system of equations (34)-(35).

Lemma 1 Suppose that Assumption 1 holds true. The equation $q(x, y)=0$ for $x>0$ defines uniquely a strictly increasing $C^{1}$ function $\left.G:\right] y_{0}, y_{\infty}[\rightarrow] 0, \infty[$, which satisfies

$$
\begin{aligned}
& \left.x^{\dagger}(y)<G(y) \text { for all } y \in\right] y_{0}, y_{\infty}\left[, \quad \lim _{y \downarrow y_{0}} G(y)=0 \text {, if } y_{0}>0,\right. \text { and } \\
& \lim _{y \uparrow y_{\infty}} G(y)=\infty,
\end{aligned}
$$

where $x^{\dagger}$ is defined by (22). Furthermore, the function A given by (37) is well-defined and real-valued, and there exists a constant $C_{1}>0$ such that

$$
\left.0<A(y) G^{n}(y) \leq C_{1} \Psi(y)\left[1+G^{n-\vartheta}(y)\right] \text { for all } y \in\right] y_{0}, y_{\infty}[
$$

where the decreasing function $\Psi$ and the constant $\vartheta>0$ are as in (21), and

$$
g^{-1}(x)+\left[1+g^{-1}(x)\right] G^{n-\vartheta}\left(g^{-1}(x)\right) \leq C_{1}\left[1+x^{n-\vartheta}\right] \text { for all } x>x_{0},
$$

where $g^{-1}$ is the inverse of the strictly increasing function $g$ that is defined by

$$
\left.g(y)=e^{c y} G(y), \text { for } y \in\right] y_{0}, y_{\infty}[\text {. }
$$

Remark 2 The last limit in (38) implies that, under the optimal strategy, if $\bar{y}<\infty$, then the maximal capacity level $\bar{y}$ is never reached. This result is due to the assumption that the function $y^{\dagger}$ appearing in Assumption 1 is such that $y^{\dagger}(\chi)<\lim _{x \rightarrow \infty} y^{\dagger}(x) \equiv$ $y_{\infty} \leq \bar{y}$ for all $\left.\chi \in\right] x_{0}, \infty$ [. Our analysis could be trivially modified to allow for the possibility that $\bar{y}<\infty$ and $\lim _{y \uparrow \bar{y}} G(y)<\infty$, which would give rise to the situation where the maximal capacity level $\bar{y}$ is reached in finite time with strictly positive probability. Such a relaxation would simply involve allowing for the strictly increasing function $y^{\dagger}$ to be such that $\lim _{x \rightarrow \infty} y^{\dagger}(x) \equiv y_{\infty}>\bar{y}$. However, we have opted against such a relaxation because this would complicate the notation and the proof of Lemma 1 substantially.

Example 2 Suppose that $h$ is a Cobb-Douglas function given by (23) in Example 1. In this case, we can check that

$$
\left.G(y)=\left[\frac{r K(\alpha-m)}{-m} \frac{y^{1-\beta}}{\beta-\alpha c y}\right]^{1 / \alpha}, \quad \text { for } y \in\right] y_{0}, y_{\infty}[\equiv] 0, \beta / c \alpha[.
$$


Figures 2 and 3 illustrate this example.

To complete the construction of the solution $w$ to the HJB equation (24) that identifies with the problem's value function $v$, we note that there exists a mapping $z: \mathcal{I} \rightarrow \mathbb{R}_{+}$such that

$z(x, y) \in]\left(y_{0}-y\right)^{+}, y_{\infty}-y\left[\right.$ and $x e^{-c z(x, y)}=G(y+z(x, y))$ for all $(x, y) \in \mathcal{I}$.

Indeed, this claim follows immediately from the calculations

$$
\begin{aligned}
& \lim _{z \uparrow y_{\infty}-y}\left[x e^{-c z}-G(y+z)\right]=-\infty \\
& \left.\frac{\partial}{\partial z}\left[x e^{-c z}-G(y+z)\right]=-c x e^{-c z}-G^{\prime}(y+z)<0, \text { for } z \in\right]\left(y_{0}-y\right)^{+}, y_{\infty}-y[\text {, } \\
& \lim _{z \downarrow\left(y_{0}-y\right)^{+}}\left[x e^{-c z}-G(y+z)\right]=\left\{\begin{array}{ll}
x e^{-c\left(y_{0}-y\right)}-\lim _{u \downarrow y_{0}} G(u), & \text { if } y \leq y_{0}, \\
x-G(y), & \text { if } y>y_{0}
\end{array}\right\}>0,
\end{aligned}
$$

in which, we have used (38) and the fact that $G$ is increasing. We prove the following result in Appendix 1.

Lemma 2 Suppose that Assumption 1 holds true. The function $w$ defined by

$$
w(x, y)= \begin{cases}R(x, y), & \text { if }(x, y) \in \mathcal{W} \cap\left(\mathbb{R}_{+} \times\left[y_{\infty}, \bar{y}\right]\right), \\ A(y) x^{n}+R(x, y), & \text { if }(x, y) \in \mathcal{W} \cap\left(\mathbb{R}_{+} \times\left[y_{0}, y_{\infty}[),\right.\right. \\ w\left(x e^{-c z(x, y)}, y+z(x, y)\right)-K z(x, y), & \text { if }(x, y) \in \mathcal{I},\end{cases}
$$

where $A$ is defined by (37) and $z$ is given by (43), is a $C^{2,1}$ solution to the HJB equation (24). Furthermore, the function $w(\cdot, y)$ is increasing and there exists a constant $C_{2}>0$ such that

$$
\begin{array}{r}
-C_{2}(1+y) \leq w(x, y) \text { for all }(x, y) \in \mathcal{S}, \\
\left.w(G(y), y) \leq C_{2}[\Psi(y)+y]\left[1+G^{n-\vartheta}(y)\right] \text { for all } y \in\right] y_{0}, y_{\infty}[,
\end{array}
$$

where the decreasing function $\Psi$ is as in (20)-(21).

We can now establish the main result of the paper.

Theorem 1 Suppose that Assumption 1 holds true. The value function $v$ of the control problem formulated in Sect. 2 identifies with the solution $w$ to the HJB equation (24) given by (44) in Lemma 2 and the optimal capacity expansion strategy $\zeta^{\star}$ is given by

$$
\zeta_{t}^{\star}=\left\{\begin{array}{ll}
0, & \text { if } y>y_{0} \text { and } e^{c y} \sup _{0 \leq s \leq t} X_{s}^{0} \leq \bar{g}(y), \\
g^{-1}\left(e^{c y} \sup _{0 \leq s \leq t} X_{s}^{0}\right), & \text { if } y<y_{\infty} \text { and } e^{c y} \sup _{0 \leq s \leq t} X_{s}^{0}>\bar{g}(y),
\end{array} \quad \text { for } t>0,\right.
$$


where

$$
\bar{g}(y)= \begin{cases}0, & \text { if } y_{0}>0 \text { and } y \leq y_{0}, \\ g(y), & \text { if } y \in] y_{0}, y_{\infty}[, \\ \infty, & \text { if } y \in\left[y_{\infty}, \bar{y}\right] \cap \mathbb{R}_{+},\end{cases}
$$

$g$ is defined by (41), and $X^{0}$ is the geometric Brownian motion given by (1).

Proof Fix any initial condition $(x, y) \in \mathcal{S}$ and any admissible strategy $\zeta \in \mathcal{A}$. In view of Itô-Tanaka-Meyer's formula and the left-continuity of the processes $X, Y$, we can see that

$$
\begin{array}{rl}
e^{-r T} & w\left(X_{T+}, Y_{T+}\right) \\
= & w(x, y)+\int_{0}^{T} e^{-r t}\left[\sigma^{2} X_{t}^{2} w_{x x}\left(X_{t}, Y_{t}\right)+b X_{t} w_{x}\left(X_{t}, Y_{t}\right)-r w\left(X_{t}, Y_{t}\right)\right] d t \\
& +\int_{[0, T]}\left[w_{y}\left(X_{t}, Y_{t}\right)-c X_{t} w_{x}\left(X_{t}, Y_{t}\right)\right] d \zeta_{t}^{c}+M_{T} \\
& +\sum_{0 \leq t \leq T} e^{-r t}\left[w\left(X_{t+}, Y_{t+}\right)-w\left(X_{t}, Y_{t}\right)\right]
\end{array}
$$

where

$$
M_{T}=\sqrt{2} \sigma \int_{0}^{T} e^{-r t} X_{t} w_{x}\left(X_{t}, Y_{t}\right) d W_{t} .
$$

Combining this calculation with the observation that

$$
\begin{aligned}
& w\left(X_{t+}, Y_{t+}\right)-w\left(X_{t}, Y_{t}\right) \\
& \stackrel{(7)}{=} \int_{0}^{\Delta \zeta_{t}} \frac{d w\left(X_{t} e^{-c s}, Y_{t}+s\right)}{d s} d s, \\
& \quad=\int_{0}^{\Delta \zeta_{t}}\left[w_{y}\left(X_{t} e^{-c s}, Y_{t}+s\right)-c X_{t} e^{-c s} w_{x}\left(X_{t} e^{-c s}, Y_{t}+s\right)\right] d s,
\end{aligned}
$$

we obtain

$$
\begin{aligned}
& \int_{0}^{T} e^{-r t} h\left(X_{t}, Y_{t}\right) d t-K \int_{[0, T]} e^{-r t} d \zeta_{t}+e^{-r T} w\left(X_{T+}, Y_{T+}\right) \\
& =w(x, y)+\int_{0}^{T} e^{-r t}\left[\sigma^{2} X_{t}^{2} w_{x x}\left(X_{t}, Y_{t}\right)+b X_{t} w_{x}\left(X_{t}, Y_{t}\right)-r w\left(X_{t}, Y_{t}\right)\right. \\
& \left.\quad+h\left(X_{t}, Y_{t}\right)\right] d t+\int_{[0, T]}\left[w_{y}\left(X_{t}, Y_{t}\right)-c X_{t} w_{x}\left(X_{t}, Y_{t}\right)-K\right] d \zeta_{t}^{c}+M_{T} \\
& \quad+\sum_{0 \leq t \leq T} e^{-r t} \int_{0}^{\Delta \zeta_{t}}\left[w_{y}\left(X_{t} e^{-c s}, Y_{t}+s\right)-c X_{t} e^{-c s} w_{x}\left(X_{t} e^{-c s}, Y_{t}+s\right)-K\right] d s
\end{aligned}
$$


Since $w$ satisfies the HJB equation (24), it follows that

$$
\int_{0}^{T} e^{-r t} h\left(X_{t}, Y_{t}\right) d t-K \int_{[0, T]} e^{-r t} d \zeta_{t}+e^{-r T} w\left(X_{T+}, Y_{T+}\right) \leq w(x, y)+M_{T}
$$

In view of the integration by parts formula and (2), we can see that

$$
e^{-r T} Y_{T_{+}}-y=-r \int_{0}^{T} e^{-r t} Y_{t} d t+\int_{[0, T]} e^{-r t} d \zeta_{t}
$$

This identity, the admissibility condition (8) in Definition 1 and the monotone convergence theorem imply that

$$
\begin{aligned}
\mathbb{E}\left[\int_{0}^{\infty} e^{-r t} Y_{t} d t\right] & =\lim _{T \rightarrow \infty} \mathbb{E}\left[\int_{0}^{T} e^{-r t} Y_{t} d t\right] \\
& \leq \lim _{T \rightarrow \infty}\left(\frac{y}{r}+\frac{1}{r} \mathbb{E}\left[\int_{[0, T]} e^{-r t} d \zeta_{t}\right]\right) \\
& =\frac{y}{r}+\frac{1}{r} \mathbb{E}\left[\int_{[0, \infty[} e^{-r t} d \zeta_{t}\right] \\
& <\infty
\end{aligned}
$$

which implies that

$$
\liminf _{T \rightarrow \infty} \mathbb{E}\left[e^{-r T} Y_{T+}\right]=0
$$

The lower bound in (20), the estimate (45) and (52) imply that

$$
\begin{aligned}
& \int_{0}^{T} e^{-r t} h\left(X_{t}, Y_{t}\right) d t-K \int_{[0, T]} e^{-r t} d \zeta_{t}+e^{-r T} w\left(X_{T+}, Y_{T+}\right) \\
& \quad \geq-C_{0} \int_{0}^{T} e^{-r t}\left(1+Y_{t}\right) d t-K \int_{[0, T]} e^{-r t} d \zeta_{t}-C_{2} e^{-r T}\left(1+Y_{T+}\right) \\
& \quad \geq-C_{0} \int_{0}^{T} e^{-r t}\left(1+Y_{t}\right) d t-\left(K+C_{2}\right) \int_{[0, T]} e^{-r t} d \zeta_{t}-C_{2}(1+y) \\
& \quad \geq-\left(\frac{C_{0}}{r}+C_{2}+C_{2} y\right)-C_{0} \int_{0}^{\infty} e^{-r t} Y_{t} d t-\left(K+C_{2}\right) \int_{[0, \infty[} e^{-r t} d \zeta_{t}
\end{aligned}
$$

The admissibility condition (8) and (53) imply that the random variable on the righthand side of these inequalities has finite expectation. Combining this observation with (51), we can see that $\mathbb{E}\left[\inf _{T \geq 0} M_{T}\right]>-\infty$. Therefore, the stochastic integral $M$ is a supermartingale and $\mathbb{E}\left[M_{T}\right] \leq 0$ for all $T>0$. Furthermore, Fatou's lemma implies that 


$$
J_{x, y}(\zeta) \leq \liminf _{T \rightarrow \infty} \mathbb{E}\left[\int_{0}^{T} e^{-r t} h\left(X_{t}, Y_{t}\right) d t-K \int_{[0, T]} e^{-r t} d \zeta_{t}\right]
$$

Taking expectations in (51) and passing to the limit, we obtain

$$
J_{x, y}(\zeta) \leq w(x, y)+\liminf _{T \rightarrow \infty} e^{-r T} \mathbb{E}\left[-w\left(X_{T+}, Y_{T+}\right)\right]
$$

The inequality $J_{x, y}(\zeta) \leq w(x, y)$ now follows because the estimate (45) implies that

$$
\liminf _{T \rightarrow \infty} e^{-r T} E\left[-w\left(X_{T+}, Y_{T+}\right)\right] \leq \lim _{T \rightarrow \infty} C_{2} e^{-r T}+C_{2} \liminf _{T \rightarrow \infty} e^{-r T} E\left[Y_{T+}\right] \stackrel{(54)}{=} 0 .
$$

Thus, we have proved that $v(x, y) \leq w(x, y)$.

To prove the reverse inequality and establish the optimality of the process $\zeta^{\star}$ given by (47), we first consider the possibility that $\left[y_{\infty}, \bar{y}\right] \cap \mathbb{R}_{+} \neq \emptyset$ and $y \in\left[y_{\infty}, \bar{y}\right]$. In this case, $\zeta_{t}^{\star}=0$ for all $t \geq 0$, and

$$
J_{x, y}\left(\zeta^{\star}\right)=\mathbb{E}\left[\int_{0}^{\infty} e^{-r t} h\left(X_{t}^{0}, y\right) d t\right] \stackrel{(29),(81)}{=} R(x, y) \stackrel{(44)}{=} w(x, y),
$$

which establish the required claims.

In the rest of the proof, we assume that $y<y_{\infty}$. In this case,

$$
Y_{t}^{\star}= \begin{cases}y, & \text { if } y \in] y_{0}, y_{\infty}\left[\text { and } e^{c y} \sup _{0 \leq s \leq t} X_{s}^{0} \leq \bar{g}(y),\right. \\ g^{-1}\left(e^{c y} \sup _{0 \leq s \leq t} X_{s}^{0}\right), & \text { if } e^{c y} \sup _{0 \leq s \leq t} X_{s}^{0}>\bar{g}(y),\end{cases}
$$

for all $t>0$, and, apart from a possible initial jump of size $\left(g^{-1}\left(e^{c y} x\right)-y\right)^{+}$at time 0 , the process $\left(e^{c y} X^{0}, Y^{\star}\right)$ is reflecting in the free-boundary $g$ in the positive direction. In particular,

$Y_{t}^{\star} \in\left[y_{0}, y_{\infty}\left[, \quad e^{c y} X_{t}^{0} \leq g\left(Y_{t}^{\star}\right) \quad\right.\right.$ and $\quad \zeta_{t}^{\star}-\zeta_{0}^{\star}=\int_{] 0, t[} \mathbf{1}_{\left\{e^{c y} X_{s}^{0}=g\left(Y_{s}^{\star}\right)\right\}} d \zeta_{s}^{\star}$ for all $t>0$

In view of (7) and the definition (41) of $g$, we can see that

$$
e^{c y} X_{t}^{0} \leq g\left(Y_{t}^{\star}\right) \Leftrightarrow X_{t}^{\star} \leq G\left(Y_{t}^{\star}\right) \quad \text { and } \quad\left\{e^{c y} X_{t}^{0}=g\left(Y_{t}^{\star}\right)\right\}=\left\{X_{t}^{\star}=G\left(Y_{t}^{\star}\right)\right\} \text {, }
$$

where $X^{\star}$ is the solution to (4) given by (7). It follows that the process $\left(X^{\star}, Y^{\star}\right)$ satisfies 
$Y_{t}^{\star} \in\left[y_{0}, y_{\infty}\left[, \quad X_{t}^{\star} \leq G\left(Y_{t}^{\star}\right)\right.\right.$ and $\zeta_{t}^{\star}-\zeta_{0}^{\star}=\int_{] 0, t[} \mathbf{1}_{\left\{X_{s}^{\star}=G\left(Y_{s}^{\star}\right)\right\}} d \zeta_{s}^{\star}$ for all $t>0$

Since the function $g$ is strictly increasing, $\zeta_{0}^{\star}>0$ if and only if $x e^{c y}>g(y) \stackrel{(41)}{=}$ $e^{c y} G(y)$. Therefore,

$$
\zeta_{0}^{\star}=\left(g^{-1}\left(e^{c y} x\right)-y\right)^{+}>0 \text { if and only if }(x, y) \in \mathcal{I} \text {. }
$$

Furthermore, given any $(x, y) \in \mathcal{I}$, we note that

$$
z=g^{-1}\left(x e^{c y}\right)-y \Leftrightarrow x e^{c y}=e^{c(y+z)} G(y+z) \Leftrightarrow x e^{-c z}=G(y+z),
$$

which implies that $\zeta_{0}^{\star}=z(x, y)$, where the function $z$ is given by (43). It follows that

$$
w\left(X_{0+}^{\star}, Y_{0+}^{\star}\right)-w(x, y)=w\left(x e^{-c z(x, y)}, y+z(x, y)\right)-w(x, y) \stackrel{(44)}{=} K z(x, y)
$$

In light of (56)-(58) and the construction of the solution $w$ to the HJB equation (24), we can see that (50) implies that

$$
\int_{0}^{T} e^{-r t} h\left(X_{t}^{\star}, Y_{t}^{\star}\right) d t-K \int_{[0, T]} e^{-r t} d \zeta_{t}^{\star}+e^{-r T} w\left(X_{T}^{\star}, Y_{T}^{\star}\right)=w(x, y)+M_{T}^{\star}
$$

for all $T>0$, where the local martingale $M^{\star}$ is defined as in (49).

To show that $\zeta^{\star}$ is indeed admissible, we use (40) and (55) to calculate

$$
Y_{t}^{\star}=y \mathbf{1}_{\left\{Y_{t}^{\star}=y\right\}}+g^{-1}\left(e^{c y} \sup _{0 \leq s \leq t} X_{s}^{0}\right) \mathbf{1}_{\left\{Y_{t}^{\star}>y\right\}} \leq y+C_{1}+C_{1} e^{c(n-\vartheta) y}\left(\sup _{0 \leq s \leq t} X_{s}^{0}\right)^{n-\vartheta}
$$

Combining these inequalities with the first estimate in (76), we can see that

$$
\lim _{T \rightarrow \infty} \mathbb{E}\left[e^{-r T} Y_{T}^{\star}\right]=0 \text { and } \mathbb{E}\left[\int_{0}^{\infty} e^{-r t} Y_{t}^{\star} d t\right]<\infty
$$

It follows that

$$
\begin{aligned}
\mathbb{E}\left[\int_{[0, \infty[} e^{-r t} d \zeta_{t}^{\star}\right] & =\lim _{T \rightarrow \infty} \mathbb{E}\left[\int_{[0, T]} e^{-r t} d \zeta_{t}^{\star}\right] \\
& \stackrel{(52)}{=} \lim _{T \rightarrow \infty}\left(\mathbb{E}\left[e^{-r T} Y_{T}^{\star}\right]+r \mathbb{E}\left[\int_{0}^{T} e^{-r t} Y_{t}^{\star} d t\right]-y\right) \\
& <\infty
\end{aligned}
$$

which proves that $\zeta^{\star} \in \mathcal{A}$. 
To proceed further, we note that the inequality in (56), the fact that $w(\cdot, y)$ is increasing and the bound given by (46) imply that, given any $t>0$,

$$
\begin{aligned}
w\left(X_{t}^{\star}, Y_{t}^{\star}\right) & \leq w\left(G\left(Y_{t}^{\star}\right), Y_{t}^{\star}\right) \\
& \leq C_{2}\left[\Psi\left(Y_{t}^{\star}\right)+Y_{t}^{\star}\right]\left[1+G^{n-\vartheta}\left(Y_{t}^{\star}\right)\right] \leq C_{2}\left[\Psi\left(Y_{0+}\right)+Y_{t}^{\star}\right]\left[1+G^{n-\vartheta}\left(Y_{t}^{\star}\right)\right],
\end{aligned}
$$

the last inequality following because $\Psi$ is decreasing. Also, (20) and (56) imply that

$$
h\left(X_{t}^{\star}, Y_{t}^{\star}\right) \leq C_{0}\left(1+Y_{t}^{\star}\right)\left(1+X_{t}^{\star n-\vartheta}\right) \leq C_{0}\left(1+Y_{t}^{\star}\right)\left[1+G^{n-\vartheta}\left(Y_{t}^{\star}\right)\right] .
$$

The estimate (40) and (55) imply that

$$
\begin{aligned}
(1+ & \left.Y_{t}^{\star}\right) G^{n-\vartheta}\left(Y_{t}^{\star}\right) \\
= & (1+y) G^{n-\vartheta}(y) \mathbf{1}_{\left\{Y_{t}^{\star}=y\right\}} \\
& +\left[1+g^{-1}\left(e^{c y} \sup _{0 \leq s \leq t} X_{s}^{0}\right)\right] G^{n-\vartheta}\left(g^{-1}\left(e^{c y} \sup _{0 \leq s \leq t} X_{s}^{0}\right)\right) \mathbf{1}_{\left\{Y_{t}^{\star}>y\right\}} \\
& \leq(1+y) G^{n-\vartheta}(y) \mathbf{1}_{\left\{y>y_{0}\right\}}+C_{1}+C_{1} e^{c(n-\vartheta) y}\left(\sup _{0 \leq s \leq t} X_{s}^{0}\right)^{n-\vartheta} .
\end{aligned}
$$

It follows that there exists a constant $C_{3}=C_{3}(y)$ such that

$$
\begin{gathered}
w\left(X_{t}^{\star}, Y_{t}^{\star}\right) \leq C_{3}\left[1+\left(\sup _{0 \leq s \leq t} X_{s}^{0}\right)^{n-\vartheta}\right] \text { and } \\
h\left(X_{t}^{\star}, Y_{t}^{\star}\right) \leq C_{3}\left[1+\left(\sup _{0 \leq s \leq t} X_{s}^{0}\right)^{n-\vartheta}\right]
\end{gathered}
$$

for all $t>0$. These inequalities and the estimates (76) imply that

$$
\begin{aligned}
& \mathbb{E}\left[\sup _{T>0}\left(\int_{0}^{T} e^{-r t} h\left(X_{t}^{\star}, Y_{t}^{\star}\right) d t+e^{-r T} w\left(X_{T}^{\star}, Y_{T}^{\star}\right)\right)\right] \\
& \leq C_{3}\left(\frac{(1+r)}{r}+\int_{0}^{\infty} \mathbb{E}\left[e^{-r t}\left(\sup _{0 \leq s \leq t} X_{s}^{0}\right)^{n-\vartheta}\right] d t\right. \\
& \left.\quad+\mathbb{E}\left[\sup _{T>0} e^{-r T}\left(\sup _{0 \leq s \leq T} X_{s}^{0}\right)^{n-\vartheta}\right]\right) \\
& <\infty
\end{aligned}
$$


and

$$
\liminf _{T \rightarrow \infty} e^{-r T} \mathbb{E}\left[-w\left(X_{T}^{\star}, Y_{T}^{\star}\right)\right] \geq-C_{3} \lim _{T \rightarrow \infty} e^{-r T}\left(1+\mathbb{E}\left[\left(\sup _{0 \leq s \leq T} X_{s}^{0}\right)^{n-\vartheta}\right]\right)=0 .
$$

In view of (59) and (61), we can see that $\mathbb{E}\left[\sup _{T>0} M_{T}^{\star}\right]<\infty$. Therefore, the stochastic integral $M^{\star}$ is a submartingale and $\mathbb{E}\left[M_{T}^{\star}\right] \geq 0$ for all $T>0$. Furthermore, Fatou's lemma implies that

$$
J_{x, y}\left(\zeta^{\star}\right) \geq \limsup _{T \rightarrow \infty} \mathbb{E}\left[\int_{0}^{T} e^{-r t} h\left(X_{t}^{\star}, Y_{t}^{\star}\right) d t-K \int_{[0, T]} e^{-r t} d \zeta_{t}^{\star}\right] .
$$

In view of these observations and (62), we can take expectations in (59) and pass to the limit to obtain

$$
J_{x, y}\left(\zeta^{\star}\right) \geq w(x, y)+\limsup _{T \rightarrow \infty} e^{-r T} \mathbb{E}\left[-w\left(X_{T}^{\star}, Y_{T}^{\star}\right)\right] \geq w(x, y) .
$$

This result and the inequality $v(x, y) \leq w(x, y)$ that we have proved above, imply that $v(x, y)=w(x, y)$ and that $\zeta^{\star}$ is optimal.

Acknowledgements We thank an anonymous referee for constructive suggestions that improved the paper.

Open Access This article is distributed under the terms of the Creative Commons Attribution 4.0 International License (http://creativecommons.org/licenses/by/4.0/), which permits unrestricted use, distribution, and reproduction in any medium, provided you give appropriate credit to the original author(s) and the source, provide a link to the Creative Commons license, and indicate if changes were made.

\section{Appendix 1: Proof of Lemmas 1 and 2}

Proof of Lemma 1. Given any $y \in] y_{0}, y_{\infty}[$, we observe that

$$
\frac{\partial}{\partial x} q(x, y)=x^{-m-1} H(x, y) \begin{cases}<0, & \text { for all } x \in] 0, x^{\dagger}(y)[, \\ =0, & \text { for all } x=x^{\dagger}(y), \\ >0, & \text { for all } x>x^{\dagger}(y),\end{cases}
$$

where $x^{\dagger}$ is defined by (22) in Assumption 1. Also, we note that (17) and (18) in Assumption 1 imply that there exist constants $\varepsilon_{1}=\varepsilon_{1}(y)$ and $x_{1}=x_{1}(y)>x^{\dagger}(y)$ such that $H(x, y) \geq \varepsilon_{1}$ for all $x \geq x_{1}$. Given such a choice of constants, we calculate

$$
\begin{aligned}
\lim _{x \rightarrow \infty} q(x, y) & =\lim _{x \rightarrow \infty}\left[q\left(x_{1}, y\right)+\int_{x_{1}}^{x} s^{-m-1} H(s, y) d s\right] \\
& \geq \lim _{x \rightarrow \infty}\left[q\left(x_{1}, y\right)+\frac{\varepsilon_{1}}{m} x_{1}^{-m}-\frac{\varepsilon_{1}}{m} x^{-m}\right] \\
& =\infty
\end{aligned}
$$


because $m<0$. Combining these observations with the fact that $q(0, y)=0$, we can see that the equation $q(x, y)=0$ for $x>0$ has a unique solution $G(y)>x^{\dagger}(y)$ for all $y \in] y_{0}, y_{\infty}[$, and that $G$ satisfies (38).

To see that the function $G:] y_{0}, y_{\infty}[\rightarrow] 0, \infty\left[\right.$ is $C^{1}$ and strictly increasing, we differentiate the identity $q(G(y), y)=0$ with respect to $y$ to obtain

$$
\dot{G}(y)=-G^{m+1}(y) H^{-1}(G(y), y) \int_{0}^{G(y)} s^{-m-1} H_{y}(s, y) d s>0,
$$

the inequality following from (19) in Assumption 1.

To establish (40), we note that

$$
\lim _{y \downarrow y_{0}} G^{n-\vartheta}(y)=e^{-c(n-\vartheta) y_{0}} \lim _{y \downarrow y_{0}} g^{n-\vartheta}(y) \leq \lim _{y \downarrow y_{0}} g^{n-\vartheta}(y)
$$

and

$$
\begin{aligned}
0 & \leq \lim _{y \uparrow y_{\infty}}(1+y) g^{-n+\vartheta}(y) \leq \lim _{y \uparrow y_{\infty}}(1+y) G^{n-\vartheta}(y) g^{-n+\vartheta}(y) \\
& =\lim _{y \uparrow y_{\infty}}(1+y) e^{-c(n-\vartheta) y}<\infty,
\end{aligned}
$$

where we have used (38) and the facts that $G$ is increasing and $n-\vartheta>0$. Combining these inequalities with the fact that $G$ and $g$ are continuous increasing functions with the same domain $] y_{0}, y_{\infty}$ [, we can see that there exists a constant $C_{1}>0$ such that

$$
\left.1+y+(1+y) G^{n-\vartheta}(y) \leq C_{1}\left[1+g^{n-\vartheta}(y)\right] \text { for all } y \in\right] y_{0}, y_{\infty}[
$$

For $x>x_{0}$ and $y=g^{-1}(x)$, this inequality implies the estimate in (40).

In view of (21) and the fact that the functions $G,-\Psi$ are increasing, we can see that, given any $y \in] y_{0}, y_{\infty}[$,

$$
\begin{aligned}
A(y) G^{n}(y) & \leq \frac{e^{c n y}}{\sigma^{2}(n-m)} G^{n}(y) \int_{y}^{y_{\infty}} e^{-c n u} \Psi(u)\left[\frac{1}{n} G^{-n}(u)+\frac{1}{\vartheta} G^{-\vartheta}(u)\right] d u \\
& \leq \frac{e^{c n y}}{\sigma^{2}(n-m)}\left[\frac{1}{n} \int_{y}^{y_{\infty}} e^{-c n u} \Psi(u) d u+\frac{1}{\vartheta} G^{n-\vartheta}(y) \int_{y}^{y_{\infty}} e^{-c n u} \Psi(u) d u\right] \\
& \leq \frac{1}{\sigma^{2}(n-m)} \Psi(y)\left[\frac{1}{c n^{2}}+\frac{1}{c n \vartheta} G^{n-\vartheta}(y)\right],
\end{aligned}
$$

which implies (39). Finally, the strict positivity of $A$ follows from (17) and the inequality in (38).

Proof of Lemma 2. In view of its construction, we will prove that $w$ is $C^{2,1}$ if we show that $w_{y}, w_{x}$ and $w_{x x}$ are continuous along the free-boundary $G$. To this end, we consider any $(x, y) \in \mathcal{I}$, we recall the definition (44) of $w$ and the definition (43) of $z$, and we use (30)-(31) to calculate 


$$
\begin{aligned}
w_{y}(x, y) Z= & \frac{\partial}{\partial y}\left[w\left(x e^{-c z(x, y)}, y+z(x, y)\right)-K z(x, y)\right] \\
= & w_{y}\left(x e^{-c z(x, y)}, y+z(x, y)\right)+\left[w_{y}\left(x e^{-c z(x, y)}, y+z(x, y)\right)\right. \\
& \left.-c x e^{-c z(x, y)} w_{x}\left(x e^{-c z(x, y)}, y+z(x, y)\right)-K\right] z_{y}(x, y) \\
= & w_{y}\left(x e^{-c z(x, y)}, y+z(x, y)\right) \\
w_{x}(x, y)= & \frac{\partial}{\partial x}\left[w\left(x e^{-c z(x, y)}, y+z(x, y)\right)-K z(x, y)\right] \\
= & w_{x}\left(x e^{-c z(x, y)}, y+z(x, y)\right) e^{-c z(x, y)} \\
& +\left[w_{y}\left(x e^{-c z(x, y)}, y+z(x, y)\right)\right. \\
& \left.-c x e^{-c z(x, y)} w_{x}\left(x e^{-c z(x, y)}, y+z(x, y)\right)-K\right] z_{x}(x, y) \\
= & w_{x}\left(x e^{-c z(x, y)}, y+z(x, y)\right) e^{-c z(x, y)}
\end{aligned}
$$

and

$$
\begin{aligned}
w_{x x}(x, y)= & \frac{\partial}{\partial x}\left[w_{x}\left(x e^{-c z(x, y)}, y+z(x, y)\right) e^{-c z(x, y)}\right] \\
= & w_{x x}\left(x e^{-c z(x, y)}, y+z(x, y)\right) e^{-2 c z(x, y)} \\
& +\left[w_{x y}\left(x e^{-c z(x, y)}, y+z(x, y)\right)\right. \\
& -c x e^{-c z(x, y)} w_{x x}\left(x e^{-c z(x, y)}, y+z(x, y)\right) \\
& \left.-c w_{x}\left(x e^{-c z(x, y)}, y+z(x, y)\right)\right] e^{-c z(x, y)} z_{x}(x, y) \\
= & w_{x x}\left(x e^{-c z(x, y)}, y+z(x, y)\right) e^{-2 c z(x, y)}
\end{aligned}
$$

These calculations imply the required continuity results because $\lim _{n \rightarrow \infty} z\left(x_{n}, y_{n}\right)=$ 0 for every convergent sequence $\left(x_{n}, y_{n}\right)$ in $\mathcal{I}$ such that $\lim _{n \rightarrow \infty} x_{n}=\lim _{n \rightarrow \infty} G\left(y_{n}\right)$.

To prove (45)-(46), we note that the bounds of $h$ in (20), the definition (29) of $R$ and the identity $\sigma^{2} m n=-r$ imply that

$$
-\frac{C_{0}}{r}(1+y) \leq R(x, y) \leq C_{0}(1+y)\left[\frac{1}{r}+\frac{1}{\sigma^{2}(n-m-\vartheta) \vartheta} x^{n-\vartheta}\right] .
$$

The lower of these bounds and the positivity of $A$ (see (39)) imply that

$$
-\frac{C_{0}}{r}(1+y) \leq A(y) x^{n}+R(x, y)=w(x, y) \text { for all }(x, y) \in \mathcal{W} .
$$

In light of (14) and (82) in Appendix 2, we can see that $R(\cdot, y)$ is increasing for all $y \in[0, \bar{y}] \cap \mathbb{R}$. Combining this observation with the inequalities $A>0$ and $n>0$, we deduce that $w_{x}(x, y) \geq 0$ for all $(x, y) \in \mathcal{W}$. This result, (43) and (65) imply that $w(\cdot, y)$ is increasing for all $y \in[0, \bar{y}] \cap \mathbb{R}$, which, combined with (68), implies (45). Also, (46) follows immediately from (39) and the upper bound in (67). 
It remains to show that $w$ satisfies the HJB equation (24). By the construction and the $C^{2,1}$ continuity of $w$, we will achieve this if we show that

$$
\begin{gathered}
\sigma^{2} x^{2} w_{x x}(x, y)+b x w_{x}(x, y)-r w(x, y)+h(x, y) \leq 0 \text { for all }(x, y) \in \mathcal{I} \\
w_{y}(x, y)-c x w_{x}(x, y)-K \leq 0 \text { for all }(x, y) \in \mathcal{W} \cap\left(\mathbb{R}_{+} \times\right] y_{0}, \bar{y}[) .
\end{gathered}
$$

To see (69), we consider any $(x, y) \in \mathcal{I}$ and we use (44), (65)-(66) and the fact that $w$ satisfies the ODE (27) inside $\mathcal{W}$ to calculate

$$
\begin{aligned}
& \sigma^{2} x^{2} w_{x x}(x, y)+b x w_{x}(x, y)-r w(x, y)+h(x, y) \\
&= \sigma^{2}\left[x e^{-c z(x, y)}\right]^{2} w_{x x}\left(x e^{-c z(x, y)}, y+z(x, y)\right)+b\left[x e^{-c z(x, y)}\right] \\
& \times w_{x}\left(x e^{-c z(x, y)}, y+z(x, y)\right) \\
&-r w\left(x e^{-c z(x, y)}, y+z(x, y)\right)+r K z(x, y)+h(x, y) \\
&=-h\left(x e^{-c z(x, y)}, y+z(x, y)\right)+h(x, y)+r K z(x, y) \\
&=-\int_{0}^{z(x, y)}\left[\frac{\partial h\left(x e^{-c u}, y+u\right)}{\partial u}-r K\right] d u \\
& \stackrel{(13)}{=}-\int_{0}^{z(x, y)} H\left(x e^{-c u}, y+u\right) d u .
\end{aligned}
$$

These calculations, (17), (38), (43) and the continuity of $z$ imply (69).

To prove (70), we first consider the possibility that $y_{\infty}<\bar{y}$. In this case, we use the fact that $w=R$ inside $\mathcal{W} \cap\left(\mathbb{R}_{+} \times\left[y_{\infty}, \bar{y}\right]\right)$, the definition (29) of $R$, the associated expression (84) for the function $x \mapsto x R_{x}(x, y)$ and (83) to calculate

$$
\begin{aligned}
& w_{y}(x, y)-c x w_{x}(x, y)-K=R_{y}(x, y)-c x R_{x}(x, y)-K \\
& \quad=\frac{1}{\sigma^{2}(n-m)}\left[x^{m} \int_{0}^{x} s^{-m-1} H(s, y) d s+x^{n} \int_{x}^{\infty} s^{-n-1} H(s, y) d s\right] \\
& \leq 0 \quad \text { for all }(x, y) \in \mathcal{W} \cap\left(\mathbb{R}_{+} \times\left[y_{\infty}, \bar{y}[),\right.\right.
\end{aligned}
$$

the inequality following thanks to (17) in Assumption 1.

To proceed further, we note that, inside $\mathcal{W} \cap\left(\mathbb{R}_{+} \times\right] y_{0}, y_{\infty}[)$, the definition (44) of $w,(32),(34)$, calculations similar to the ones in (71) and the definition (13) of $H$ imply that

$$
\begin{aligned}
\varrho(x, y) & :=w_{y}(x, y)-c x w_{x}(x, y)-K \\
& =\frac{1}{\sigma^{2}(n-m)}\left[-x^{m} \int_{x}^{G(y)} s^{-m-1} H(s, y) d s+x^{n} \int_{x}^{G(y)} s^{-n-1} H(s, y) d s\right] .
\end{aligned}
$$


In light of (17), (38) and the fact that $m<0<n$, we can see that

$$
\begin{aligned}
\varrho_{x}(x, y)= & \frac{1}{\sigma^{2}(n-m)}\left[-m x^{m-1} \int_{x}^{G(y)} s^{-m-1} H(s, y) d s\right. \\
& \left.+n x^{n-1} \int_{x}^{G(y)} s^{-n-1} H(s, y) d s\right] \\
\geq & 0 \quad \text { for all } x \in\left[x^{\dagger}(y), G(y)\right],
\end{aligned}
$$

which, combined with the identity $\varrho(G(y), y)=0$, implies that

$$
\varrho(x, y) \leq 0 \text { for all } x \in\left[x^{\dagger}(y), G(y)\right]
$$

Also, we can use the inequality

$$
\left.\int_{x}^{G(y)} s^{-m-1} H(s, y) d s>0 \text { for all } x \in\right] 0, G(y)[,
$$

which follows from (17) in Assumption 1 and (34), to calculate

$$
\begin{aligned}
\lim _{x \downarrow 0} \varrho(x, y) & \leq \frac{1}{\sigma^{2}(n-m)} \lim _{x \downarrow 0} x^{n} \int_{x}^{G(y)} s^{-n-1} H(s, y) d s \\
& =\frac{1}{\sigma^{2}(n-m)} \lim _{x \downarrow 0} x^{n} \int_{x}^{x^{\dagger}(y)} s^{-n-1} H(s, y) d s \\
& \leq 0
\end{aligned}
$$

the inequality following from (17) and the fact that $n>0$.

Finally, we can use the fact that $m, n$ are the solutions to the quadratic equation (11) and straightforward calculations to obtain

$$
\left.\sigma^{2} x^{2} \varrho_{x x}(x, y)+b x \varrho_{x}(x, y)-r \varrho(x, y)=-H(x, y)>0 \text { for all } x \in\right] 0, x^{\dagger}(y)[\text {. }
$$

This inequality and the maximum principle imply that the function $\varrho$ has no positive maximum inside ]0, $x^{\dagger}(y)$ [, which, combined with (73)-(74), implies that $\varrho(x, y) \leq 0$ for all $y \in] y_{0}, y_{\infty}[$ and $\left.x \in] 0, G(y)\right]$, and (70) follows.

\section{Appendix 2: A Second Order Linear ODE}

In this section, we review certain results regarding the solvability of a second order linear ODE on which our analysis has been based. All of the claims that we do not prove here are standard and can be found in several references (e.g., with the exception of (76), which is proved in Merhi and Zervos [40, Lemma 1], all results can be found in Knudsen et al. [33]). 
Given a constant $\lambda$,

$$
\begin{aligned}
\mathbb{E}\left[\int_{0}^{\infty} e^{-r t}\left(X_{t}^{0}\right)^{\lambda} d t\right] & =x^{\lambda} \int_{0}^{\infty} e^{\left[\sigma^{2} \lambda^{2}+\left(b-\sigma^{2}\right) \lambda-r\right] t} \mathbb{E}\left[e^{-\sigma^{2} \lambda^{2} t+\sqrt{2} \sigma \lambda W_{t}}\right] d t \\
& = \begin{cases}\infty, & \text { if } \lambda \leq m \text { or } \lambda \geq n, \\
-x^{\lambda} /\left[\sigma^{2} \lambda^{2}+\left(b-\sigma^{2}\right) \lambda-r\right], & \text { if } \lambda \in] m, n[,\end{cases}
\end{aligned}
$$

where $X^{0}$ is the geometric Brownian motion given by (1) and $m<0<n$ are the constants defined by (12). Furthermore, for all $\lambda \in] 0, n[$, there exist constants $\varepsilon, C>0$ such that

$$
e^{-r T} \mathbb{E}\left[\left(\sup _{0 \leq t \leq T} X_{t}^{0}\right)^{\lambda}\right] \leq C x^{\lambda} e^{-\varepsilon T} \text { and } \mathbb{E}\left[\sup _{T \geq 0} e^{-r T}\left(\sup _{0 \leq t \leq T} X_{t}^{0}\right)^{\lambda}\right] \leq C x^{\lambda}
$$

for all $x>0$.

A Borel measurable function $k:] 0, \infty[\rightarrow \mathbb{R}$ satisfies

$$
\mathbb{E}\left[\int_{0}^{\infty} e^{-r t}\left|k\left(X_{t}^{0}\right)\right| d t\right]<\infty \text { for all } x>0
$$

if and only if

$$
\int_{0}^{x} s^{-m-1}|k(s)| d s+\int_{x}^{\infty} s^{-n-1}|k(s)| d s<\infty \text { for all } x>0 .
$$

In the presence of these equivalent integrability conditions, the function $R$ defined by

$$
R(x)=\frac{1}{\sigma^{2}(n-m)}\left[x^{m} \int_{0}^{x} s^{-m-1} k(s) d s+x^{n} \int_{x}^{\infty} s^{-n-1} k(s) d s\right], \quad \text { for } x>0,
$$

is a special solution to the non-homogeneous ODE

$$
\sigma^{2} x^{2} u^{\prime \prime}(x)+b x u^{\prime}(x)-r u(x)+k(x)=0
$$

that admits the probabilistic expression

$$
R(x)=\mathbb{E}\left[\int_{0}^{\infty} e^{-r t} k\left(X_{t}^{0}\right) d t\right]
$$

Furthermore,

if $k$ is increasing, then $R$ is increasing, and, if $k$ is constant, then $r R(x)=k$ for all $x>0$. 
In our analysis we have used the following result.

Lemma 3 Consider any $C^{1}$ function $\left.k:\right] 0, \infty[\rightarrow \mathbb{R}$ satisfying the equivalent integrability conditions (77)-(78) and suppose that there exists $\varepsilon>0$ such that

$\forall x<\varepsilon$, either $k^{\prime}(x) \geq 0$ or $k^{\prime}(x) \leq 0$ and $\forall x>\varepsilon^{-1}$, either $k^{\prime}(x) \geq 0$ or $k^{\prime}(x) \leq 0$.

Then

$$
x R^{\prime}(x)=\frac{1}{\sigma^{2}(n-m)}\left[x^{m} \int_{0}^{x} s^{-m} k^{\prime}(s) d s+x^{n} \int_{x}^{\infty} s^{-n} k^{\prime}(s) d s\right] \text { for all } x>0,
$$

in which expression, both integrals are well-defined and real-valued.

Proof We first note that the integrability condition (78) implies that the limits

$$
\lim _{z \downarrow 0} \int_{z}^{x} s^{-m-1} k(s) d s \text { and } \lim _{z \rightarrow \infty} \int_{x}^{z} s^{-n-1} k(s) d s
$$

exist in $\mathbb{R}$ and that

$$
\liminf _{z \downarrow 0} z^{-m}|k(z)|=0 \text { and } \liminf _{z \rightarrow \infty} z^{-n}|k(z)|=0 .
$$

To see the latter claim, suppose that $\lim \inf _{z \downarrow 0} z^{-m}|k(z)|>0$. In such a case, there exist constants $\varepsilon, z_{1}>0$ such that $z^{-m}|k(z)| \geq \varepsilon$ for all $z \leq z_{1}$. Therefore,

$$
\int_{0}^{z_{1}} s^{-m-1}|k(s)| d s \geq \varepsilon \int_{0}^{z_{1}} s^{-1} d s=\infty
$$

which contradicts (78). We can argue similarly by contradiction to prove the second limit in (85).

Using the integration by parts formula, we calculate

$x^{-m} k(x)-z^{-m} k(z)=-m \int_{z}^{x} s^{-m-1} k(s) d s+\int_{z}^{x} s^{-m} k^{\prime}(s) d s$ for all $0<z<x$.

The assumptions that we have made on $k^{\prime}$ and the monotone convergence theorem imply that the limit $\lim _{z \downarrow 0} \int_{z}^{x} s^{-m} k^{\prime}(s) d s$ exists. Therefore, we can pass to the limit as $z \downarrow 0$ in (86) to obtain

$$
x^{-m} k(x)=-m \int_{0}^{x} s^{-m-1} k(s) d s+\int_{0}^{x} s^{-m} k^{\prime}(s) d s \text { for all } x>0 .
$$

Similarly, we can see that

$$
-x^{-n} k(x)=-n \int_{x}^{\infty} s^{-n-1} k(s) d s+\int_{x}^{\infty} s^{-n} k^{\prime}(s) d s \text { for all } x>0 .
$$


The required result follows immediately from these calculations and the expression

$$
x R^{\prime}(x)=\frac{1}{\sigma^{2}(n-m)}\left[m x^{m} \int_{0}^{x} s^{-m-1} k(s) d s+n x^{n} \int_{x}^{\infty} s^{-n-1} k(s) d s\right] .
$$

\section{References}

1. Abel, B.A., Eberly, J.C.: Optimal investment with costly reversibility. Rev. Econ. Stud. 63, 581-593 (1996)

2. Alvarez, L. H. R. (2006), A general theory of optimal capacity accumulation under price uncertainty and costly reversibility, Helsinki Center of Economic Research, Working Paper

3. Alvarez, L.H.R.: Irreversible capital accumulation under interest rate uncertainty. Math. Meth. Oper. Res. 72, 249-271 (2010)

4. Arntzen, H.: Optimal choice of production capacity in a random market. Stochast. Stochast. Rep. 55, 87-120 (1995)

5. Bahlali, K., Chighoub, F., Djehiche, B., Mezerdi, B.: Optimality necessary conditions in singular stochastic control problems with nonsmooth data. J. Math. Anal. Appl. 355, 479-494 (2009)

6. Bank, P.: Optimal control under a dynamic fuel constraint. SIAM J. Control Optim. 44, 1529-1541 (2005)

7. Bather, J.A., Chernoff, H.: Sequential decisions in the control of a spaceship. Fifth Berkeley Symposium on Mathematical Statistics and Probability 3, 181-207 (1967)

8. Beneš, V.E., Shepp, L.A., Witsenhausen, H.S.: Some solvable stochastic control problems. Stochast. Stochast. Rep. 4, 39-83 (1980)

9. Chiarolla, M.B., Ferrari, G.: Identifying the free boundary of a stochastic, irreversible investment problem via the Bank-El Karoui representation theorem. SIAM J. Control Optim. 52, 1048-1070 (2014)

10. Chiarolla, M.B., Haussmann, U.G.: The optimal control of the cheap monotone follower. Stochast. Stochast. Rep. 49, 99-128 (1994)

11. Chiarolla, M.B., Haussmann, U.G.: Explicit solution of a stochastic irreversible investment problem and its moving threshold. Math. Oper. Res. 30, 91-108 (2005)

12. Chow, P.L., Menaldi, J.L., Robin, M.: Additive control of stochastic linear systems with finite horizon. SIAM J. Control. Optim. 23, 858-899 (1985)

13. Davis, M.H.A., Dempster, M.A.H., Sethi, S.P., Vermes, D.: Optimal capacity expansion under uncertainty. Adv. Appl. Prob. 19, 156-176 (1987)

14. Davis, M.H.A., Zervos, M.: A pair of explicitly solvable singular stochastic control problems. Appl. Math. Optim. 38, 327-352 (1998)

15. De Angelis, T., Federico, S., Ferrari, G.: Optimal boundary surface for irreversible investment with stochastic costs, preprint (2016)

16. De Angelis, T., Ferrari, G.: A stochastic partially reversible investment problem on a finite time-horizon: free-boundary analysis. Stochast. Process. Appl. 124, 4080-4119 (2014)

17. Dixit, A.K., Pindyck, R.S.: Investment Under Uncertainty. Princeton University Press, Princeton (1993)

18. Dufour, F., Miller, B.M.: Generalized solutions in nonlinear stochastic control problems. SIAM J. Control Optim. 40, 1724-1745 (2002)

19. Federico, S., Pham, H.: Characterization of the optimal boundaries in reversible investment problems. SIAM J. Control Optim. 52, 2180-2223 (2014)

20. Fleming, W.H., Soner, H.M.: Controlled Markov Processes and Viscosity Solutions. Springer, New York (1993)

21. Guo, X., Kaminsky, P., Tomecek, P., Yuen, M.: Optimal spot market inventory strategies in the presence of cost and price risk. Math. Meth. Oper. Res. 73, 109-137 (2011)

22. Guo, X., Pham, H.: Optimal partially reversible investments with entry decisions and general production function. Stochast. Process. Appl. 115, 705-736 (2005)

23. Guo, X., Tomecek, P.: Connections between singular control and optimal switching. SIAM J. Control. Optim. 47, 421-443 (2008) 
24. Guo, X., Tomecek, P.: A class of singular control problems and the smooth fit principle. SIAM J. Control Optim. 47, 3076-3099 (2008)

25. Guo, X., Zervos, M.: Optimal execution with multiplicative price impact. SIAM J. Financ. Math. 6, 281-306 (2015)

26. Harrison, J.M., Taksar, M.I.: Instantaneous control of Brownian motion. Math. Oper. Res. 8, 439-453 (1983)

27. Haussmann, U.G., Suo, W.: Singular optimal stochastic controls. I. Existence. SIAM J. Control Optim. 33, 916-936 (1995)

28. Haussmann, U.G., Suo, W.: Singular optimal stochastic controls. II. Dynamic programming. SIAM J. Control Optim. 33, 937-959 (1995)

29. Jack, A., Johnson, T.C., Zervos, M.: A singular control problem with application to the goodwill problem. Stochast. Process. Appl. 118, 2098-2124 (2008)

30. Jacka, S.: A finite fuel stochastic control problem. Stochastics 10, 103-113 (1983)

31. Jacka, S.: Avoiding the origin: a finite-fuel stochastic control problem. Ann. Appl. Prob. 12, 1378-1389 (2002)

32. Karatzas, I.: A class of singular stochastic control problems. Adv. Appl. Prob. 15, 225-254 (1983)

33. Knudsen, T.S., Meister, B., Zervos, M.: Valuation of investments in real assets with implications for the stock prices. SIAM J. Control Optim. 36, 2082-2102 (1998)

34. Kobila, T.Ø.: A class of solvable stochastic investment problems involving singular controls. Stochast. Stochast. Rep. 43, 29-63 (1993)

35. Løkka, A., Zervos, M.: A model for the long-term optimal capacity level of an investment project. Int. J. Theor. Appl. Financ. 14, 187-196 (2011)

36. Løkka, A., Zervos, M.: Long-term optimal investment strategies in the presence of adjustment costs. SIAM J. Control Optim. 51, 996-1034 (2013)

37. Ma, J.: On the principle of smooth fit for a class of singular stochastic control problems for diffusions. SIAM J. Control Optim. 30, 975-999 (1992)

38. Manne, A.S.: Capacity expansion and probabilistic growth. Econometrica 29, 632-649 (1961)

39. Menaldi, J.-L., Robin, M.: On some cheap control problems for diffusion processes. Trans. Am. Math. Soc. 278, 771-802 (1983)

40. Merhi, A., Zervos, M.: A model for reversible investment capacity expansion. SIAM J. Control Optim. 46, 839-876 (2007)

41. Motta, M., Sartori, C.: Finite fuel problem in nonlinear singular stochastic control. SIAM J. Control Optim. 46, 1180-1210 (2007)

42. Øksendal, A.: Irreversible investment problems. Financ. Stochast. 4, 223-250 (2000)

43. Shreve, S.E., Lehoczky, J.P., Gavers, D.P.: Optimal consumption for general diffusions with absorbing and reflecting barriers. SIAM J. Control Optim. 22, 55-75 (1984)

44. Soner, H.M., Shreve, S.E.: Regularity of the value function for a two-dimensional singular stochastic control problem. SIAM J. Control Optim. 27, 876-907 (1989)

45. Steg, J.-H.: Irreversible investment in oligopoly. Financ. Stochast. 16, 207-224 (2012)

46. Sun, M.: Singular control problems in bounded intervals. Stochast. Stochast. Rep. 21, 303-344 (1987)

47. Van Mieghem, J.A.: Commissioned paper: capacity management, investment, and hedging: review and recent developments. Manufact. Serv. Oper. Manage. 5, 269-302 (2003)

48. Wang, H.: Capacity expansion with exponential jump diffusion processes. Stochast. Stochast. Rep. 75, 259-274 (2003)

49. Zhu, H.: Generalized solution in singular stochastic control: the nondegenerate problem. Appl. Mathe. Optim. 25, 225-245 (1992) 